\title{
COVID-19 y práctica de actividad física y deporte entre mujeres adultas españolas: Contexto y recomendaciones
}

\section{COVID-19 and practice of physical activity and sport among} Spanish adult women: Context and recommendations

María Martín Rodríguez Grupo de investigación psicosocial en el deporte - Colegiada $n^{\circ} 9.333$ Facultad de Ciencias de la Actividad Física y del Deporte (INEF) Universidad Politécnica de Madrid maría.martin@upm.es

\section{Palabras clave \\ - COVID-19, \\ - Mujeres adultas, \\ - Actividad física \\ - Deporte}

\section{Key words}

- COVID-19,

- Adult women,

- Physical activity

- Sport

\section{Resumen}

Los objetivos del presente trabajo fueron resumir el conocimiento clave y recomendaciones para desarrollar acciones basadas en la evidencia vinculadas al COVID-19 y la práctica de actividad física y deporte de las mujeres adultas españolas, con especial atención a su conciliación con la maternidad y el empleo. Se comienza resumiendo sus necesidades, hábitos y barreras pre y post COVID-19, así como así específicas COVID-19 de organizaciones de referencia. Y se concluye estableciendo elementos clave y recomendaciones para re-crear mejores actividades y espacios más seguros para la práctica de las mujeres adultas.

\footnotetext{
Abstract

The objectives of the present paper were to summarize key knowledge and recommendations to develop evidence-based actions related to COVID-19 and practice of physical activity and sport of the Spanish adult women, with special focus to on combining maternity and employment. First, pre and post COVID-19 needs, habits and barriers were summarized, as well as specific proposals related to COVID-19 from reference organizations. This work concludes by summarizing key elements and recommendations in order to "re-create" better activities and safer spaces for adult women to practice in.
} 
Este trabajo surge a partir de un informe realizado a instancia de un encargo público, presentado en comparecencia como experta, para incorporar la perspectiva de género a la reconstrucción de la actividad física y el deporte postCOVID-19 en los Pactos de Cibeles del Ayuntamiento de Madrid. Para desarrollar acciones públicas basadas en la evidencia, se revisó el conocimiento disponible sobre la situación previa y el impacto del COVID-19 en la práctica de actividad física, ejercicio físico y deporte (en adelante AFD) de las mujeres adultas españolas, con especial atención a la conciliación de crianza y empleo, así como recomendaciones y hojas de ruta de referencia. Concluye el trabajo resumiendo elementos clave y recomendaciones a considerar para el desarrollo de políticas deportivas.

\section{Las mujeres adultas como población diana}

Para promover estilos de vida físicamente activos y saludables en España las mujeres adultas son un grupo clave por varias razones. La primera es que esta generación del baby boom son un grupo muy numeroso; una de cada dos personas en España se sitúa en la franja entre 30 y 64 años (Instituto Nacional de Estadística, 2020). La segunda es porque son fundamentales para reducir el sedentarismo y obesidad infanto-juvenil, impulsando en sus hijas e hijos la práctica de AFD, dado que tener modelos familiares es la principal variable predictora de práctica, siendo este efecto mayor en las mujeres (Ministerio de Cultura y Deporte, 2020). La tercera es que la práctica en un ciclo de vida anterior aumenta las posibilidades de seguir practicando (Martínez del Castillo et al., 2010; Moscoso et al., 2008), esta cuestión es fundamental en un país envejecido, ya que la vejez es territorio de mujeres (Instituto Nacional de Estadística, 2020). Por lo demás, se trata este de un grupo de población que también resulta fundamental para profesionales y organizaciones del sector fitness-wellness, al demandar significativamente más que los hombres servicios deportivos dirigidos (Espada et al., 2019; Martín et al., 2014).

\section{Su difícil conciliación de la práctica de AFD con la crianza y el empleo}

En mujeres, la edad adulta es el periodo vital donde confluyen circunstancias que limitan de manera importante su disponibilidad de tiempo libre para la práctica de AFD, entre otras, el empleo junto a las tareas de cuidado de personas y hogar, que han sido tradicionalmente atribuidas a este grupo de población. En relación a la conciliación de empleo y cuidados, aunque se ha ampliado la participación de las mujeres españolas en el mercado laboral, ellas siguen asumiendo la mayor parte de las responsabilidades familiares y domésticas. Es la denominada "doble o triple jornada o rol femenino", porque las diferencias entre hombres y mujeres en el reparto de horas entre trabajo remunerado y no remunerado evidencia el desigual reparto de las tareas de crianza, educación, cuidado de mayores, dependientes y trabajo doméstico, así como las jornadas de trabajo totales más largas que realizan éstas (Instituto de la Mujer y para la Igualdad de oportunidades, 2020b).

García y Llopis (2011) señalaron que, en España, uno de los dos momentos de abandono de la práctica de las mujeres se producía por la confluencia de maternidad y empleo. Otros autores atribuyen al triple rol femenino, entre otros, la "clara persistencia de desigualdades sociales crónicas (o "brecha deportiva") en relación al género y la edad (Moscoso y Rodríguez, 2020, 199).

En relación a las necesidades y barreras de la población adulta española Martín et al. (2014) evidenciaron que los hombres practicaban más ejercicio físico y deporte (51\% y 31\%, respectivamente), más mujeres que hombres caminaban para ejercitarse $(25,5 \%$ y $13,2 \%$, respectivamente), mientras que más mujeres que hombres, aunque no practicaban, deseaban hacerlo (23,9\% y $18 \%$, respectivamente).

Martín, Barriopedro y Espada (2020) pusieron de manifiesto que las madres empleadas representan el grupo con mayores puntuaciones en las principales barreras, como la dificultad de generar tiempo para sí mismas, el empleo y horarios incompatibles. En las madres, empleadas o no, el cuidado de su/s hija/os aparecía en mayor medida cuanto menor era la edad de las mujeres.

Aunque la subida del IVA desde 2012, que gravó Ios servicios deportivos del 10\% al 21\%, disminuyó en un tercio el porcentaje de mujeres practicantes con ayuda o dirección profesional ${ }^{1}$-es decir, en servicios deportivos organizados- (Martín, Espada y Barriopedro, 2020), el doble de ellas que de ellos practicaba con dirección profesional (Espada et al., 2019), especialmente las adultas jóvenes, si bien durante menos tiempo y en días laborables (Espada et al., 2018).

\footnotetext{
${ }^{1}$ Tipo de práctica de AFD dirigida, supervisada y orientada por profesionales con cualificación para desarrollar estas funciones de educadores/as físico deportivos/as.
} 
La confluencia de barreras de los espacios privados (déficits de corresponsabilidad) y público (déficits de una oferta de práctica conciliadora con crianza y empleo y subida del IVA), pudiera explicar la alta prevalencia de práctica autónoma (68,5\%). Ello, principalmente, en actividades consistentes en caminar por espacios urbanos al aire libre. Además, explicarían tales barreras el elevado número de mujeres adultas sedentarias que deseaban practicar deporte acudiendo a medios de ayuda o dirección profesional (68\%) en un servicio deportivo público municipal (47\%).

Las innovaciones a emprender solicitadas fueron programas de ejercicio físico en el trabajo, en el propio centro o en coordinación con instalaciones próximas. Pero la más demandada, tanto por hombres como mujeres y a emprender por su escasez, fue la coordinación de la oferta adultas e infancia, mediante programas de práctica conjuntos de adultas y menores, así como la oferta de diferentes actividades infantiles y para mujeres adultas en mismo horario y espacio, ludoteca o guardería en instalación deportiva (Martín et al., 2014). Proporcionar cuidado o actividades infantiles en el mismo tiempo que las actividades de las madres y padres también se recomienda en varias herramientas españolas y europeas (Consejo Superior de Deportes-Federación Española de Municipios y Provincias, 2011; Council of Europe, 2019a, 2019b). Así mismo, el estudio referencial From Brighton to Helsinki. Women and Sport Progress Report 1994-2014 (Fasting et al., 2014) concretaba que el desarrollo de provisión de cuidados infantiles para las personas involucradas en el deporte en diferentes roles y áreas es una de las cuatro acciones prioritarias para mejorar la participación de las mujeres en el deporte.

\section{El impacto del COVID-19 en la práctica de AFD de las mujeres adultas}

El informe del Instituto de la Mujer y para la Igualdad de oportunidades La perspectiva de género, esencial en la respuesta a la COVID-19 (2020a) reflejó como el impacto sanitario, social, familiar y económico de esta pandemia, incrementó las barreras vinculadas al cuidado y amplió otras relacionadas con empleo y la seguridad:

- Centralidad de las tareas de cuidado: A sus dificultades habituales, se ha añadido el cierre de colegios y limitación de sus redes de apoyo comunitarias y familiares. Algunas han tenido que conciliar teletrabajo y telecolegio, otras no han podido seguir trabajando.

- Sobrecarga del trabajo sanitario y de servicios esenciales: Las mujeres son la mayoría del personal sanitario, del comercio de alimentación, así como de limpieza hospitalaria y de residencias.

- Mayor pobreza y precariedad laboral: A la brecha de tasa de empleo femenino, elevada temporalidad y precariedad, hay que añadir que algunos de los sectores más afectados, como comercio, turismo y hostelería, están muy feminizados.

- Incremento del riesgo de violencia de género: Una de las manifestaciones de este impacto ha sido el incremento de consultas online y llamadas al 016 durante el confinamiento.

Castañeda et al. (2020) atribuyeron a la sobrecarga en las tareas de cuidado algunos de los cambios en los comportamientos de mujeres y hombres (entre 18 y 64 años) durante el confinamiento domiciliario. El tiempo sentado aumentó más en los hombres, que también redujeron las actividades moderadas, mientras que las mujeres las aumentaron. El caminar se redujo a la mitad en ambos y ellos más que ellas disminuyeron las actividades físicamente vigorosas, como la práctica de ejercicio físico o deporte, relacionando esto con la brecha deportiva de género. Cabe recordar que 7 de cada 10 mujeres activas partían de un hábito de práctica autónomo y que, en torno al $43 \%$ de las mujeres adultas españolas, del sedentarismo.

En hombres y mujeres adultas, García et al. (2020) evidenciaron una disminución de intensidad y cantidad en ambos sexos, así como cambios más acusados en las mujeres, desde ejercicio cardio-respiratorio y muscular a flexibilidad y neuromotor. Prevaleció la práctica autónoma en ambos, más en hombres, siendo la práctica virtual significativamente mayor entre las mujeres.

El Informe Impacto COVID-19 en Instalaciones Deportivas (VALGO- Federación Nacional de Empresarios de Instalaciones Deportivas, 2020) concreta las acciones virtuales que emprendieron las instalaciones públicas de gestión privada y centros privados. La principal clases online a través de redes, seguida de contenidos en web, Apps para móviles propias, canal Youtube y sesiones en directo por videoconferencia. Esto sugiere que quienes antes practicaban con dirección profesional tuvieron una oferta adaptada y segura, aunque el informe recoge que aproximadamente la mitad del sector fitness e instalaciones deportivas españolas presentan dificultades de supervivencia.

Castañeda et al. (2020) evidenciaron que la población menos activa incrementó la realización de actividades vigorosas y moderadas, que atribuyen a la promoción de recomendaciones, entrenamientos matutinos en la televisión pública y sesiones online. Piedra (2020) señalaba que algunas de las campañas 
que más difusión tuvieron fueron \#YoMeMuevoEnCasa y \#YoEntrenoEnCasa, desarrolladas por influencers. Partían mejor posicionadas y tuvieron mayor seguimiento las influencers Michelle Lewin, la reina del fitness en las redes, Jen Setler o Kayla Itsines que los influencers Simeon Panda o Joe Wicks. La influencer española más seguida fue Patry Jordan, con seguimientos muy superiores a los licenciados en CCAFYDE Sergio Peinado y David Marchante. Durante el COVID-19 ganó 230.000 seguidores/as en Instagram desde marzo, y su Canal de Youtube pasó de unas 20 millones de reproducciones al mes a las $150 \mathrm{mi}-$ llones en abril o 123 millones en mayo. Destacando que la mayoría de influencers carecen de titulación profesional para dirigir ejercicio físico, López, Rodríguez y Salcedo (2020) alertaban sobre el aumento de lesiones detectadas por especialistas de la medicina derivadas de una práctica sin supervisión profesional en hombres y mujeres adultas.

En la desescalada, cuando se permitió salir a los niños y niñas a pasear acompañados de una/a progenitor/a, se presentaron las aglomeraciones y el riesgo de accidentes. También acciones novedosas y adaptativas de la población, que señalaban la necesidad y oportunidad de una transformación urbana facilitadora del ejercicio y el movimiento lúdico, de práctica conjunta o coordinada adultas-infancia, en espacios más saludables (Sánchez, 2020).

\section{La crisis del COVID-19 como oportunidad para mejorar la práctica de AFD de las mujeres adultas.}

La Ejecutiva Global del Grupo internacional Mujeres y Deporte declaró la pandemia COVID-19 como una amenaza para el movimiento "mujeres en el deporte y la actividad física", emitiendo una llamada a la acción global mediante su IWG responds to COVID 19 with global 'Call to Action'. Alentaron a las organizaciones firmantes de la Brighton plus Helsinki 2014 Declaration on Women \& Sport a liderar con el ejemplo el desarrollo, recuperación y reconstrucción de una cultura que permita y valore la plena participación de las mujeres y las niñas en todos los aspectos del deporte y la actividad física. Plantean cinco áreas, a considerar para las mujeres adultas las siguientes:

Area 1 Bienestar: Regreso a la actividad física "fuera de casa" tan pronto como sea segura pero, mientras tanto, movimiento "en el hogar".

Area 2 Seguridad: "Re-crear" actividades y espacios seguros para las mujeres.

Area 3 Recursos: Planificar equitativamente recursos y cronograma de recuperación.
Area 4 Liderazgo: Inclusión de las aportaciones de las mujeres y las comunidades en el nuevo diseño organizativo para afrontar este reto.

Area 5 Estructura: "Re-imaginar" nuevas formas de funcionar que incluyan con seguridad y equidad a las mujeres y las comunidades diversas.

A nivel nacional destacar las recomendaciones, solicitudes y manifiesto del Consejo General de la Educación Física y Deportiva para una práctica más segura con dirección profesional, la exención durante el confinamiento y reducción del $21 \%$ al $4 \%$ del IVA de los servicios profesionales, así como la solicitud de que sean reconocidos como actividad esencial, bajo los mismos parámetros, facilitando espacios públicos y ayudas económicas para su adaptación y destacando sus beneficios frente al COVID-19 y bajo riesgo de contagio $(0,34 \%)$.

\section{Conclusiones y recomendaciones}

El conocimiento expuesto se resume en las siguientes cuestiones clave.

En el contexto del COVID-19, una población diana sobre la que actuar en materia deportiva son las mujeres adultas, grupo muy numeroso en España que demanda, más que los hombres, servicios fitness con dirección profesional, por lo que son fundamentales para el sector profesional. Además, son claves para impulsar estilos de vida físicamente activos en la población infanto-juvenil y mayor.

Ellas partían de una menor y peor práctica. Aproximadamente, de cada 10 practicantes 4 utilizaban una instalación deportiva, 3 se ejercitaban con dirección profesional y 7 con autonomía, principalmente caminando en espacios urbanos al aire libre. 4 de cada 10 mujeres iniciaron el confinamiento desde el sedentarismo; 2 de ellas, aunque no practicaban deseaban hacerlo.

Sus principales barreras para la práctica de AFD fueron la dificultad de generar tiempo para si mismas, el empleo y horarios, especialmente madres empleadas, junto al cuidado de su/s hija/os, principalmente las madres más jóvenes, empleadas o no. En estos grupos confluían déficits de corresponsabilidad en tareas de cuidado y de oferta de práctica conciliadora con crianza y empleo.

El impacto del COVID-19 ha sido mayor en las mujeres por el incremento de las tareas de cuidado domésticas, así como por la sobrecarga del feminizado empleo sanitario y de servicios esenciales, mayor precariedad laboral, pobreza y aumento del riesgo de sufrir violencia de género.

Durante el confinamiento las mujeres aumentaron la realización de actividades moderadas, atribui- 
das a tareas de cuidado, mientras que los hombres las disminuyeron. Ellos aumentaron más que ellas los comportamientos sedentarios. Hombres y mujeres adultas disminuyeron la cantidad e intensidad de AFD.

Las mujeres adultas encontraron en la práctica de AFD un elemento compensador y protector de su salud y bienestar durante la cuarentena. La población menos activa incrementó la realización de actividades vigorosas y moderadas. Ellas cambiaron más que ellos el tipo de actividad, desde ejercicio cardio-respiratorio y muscular a flexibilidad y neuromotor, siendo la práctica virtual significativamente mayor entre las mujeres. Podría decirse que para ellas fue una "cuar-entrena online".

Las clientas y usuarias de instalaciones deportivas tuvieron una oferta más segura y adaptada con dirección profesional, mediante clases online a través de redes, contenidos en web, aplicaciones de móvil, canales Youtube y sesiones en directo por videoconferencia.

Las influencers partían e incrementaron su seguimiento más que ellos, aunque la mayoría carece de titulación profesional para dirigir ejercicio físico. Especialistas de la medicina alertaron del aumento de lesiones en personas adultas derivadas de una práctica sin supervisión profesional. La desescalada mostró aglomeraciones y riesgo de accidentes en los espacios urbanos al aire libre.

Para desarrollar acciones basadas en la evidencia recogida, teniendo en consideración las propuestas de organizaciones de referencia se recomienda:

- Re-crear" mejores actividades y espacios de práctica de AFD, presenciales y vituales, con nuevas formas de organización participativa, para incorporar las necesidades de las mayorías sociales clave en la promoción de estilos de vida físicamente activos y saludables, como las de las madres adultas, hasta ahora escasamente tenidas en cuenta.

- Facilitar y promover una práctica de AFD más segura orientada por profesionales.

- Desarrollar nuevas formas, actividades y espacios de práctica deportiva conjunta de adultas y menores, saludable y lúdica, autónoma y dirigida.

- Promover la conciliación de la práctica diferenciada, coordinando en el mismo horario y espacio la práctica para adultas y menores, incluyendo el juego.

- Adaptar los espacios al aire libre para la práctica de AFD autónoma y dirigida por profesionales, conjunta y diferenciada adultas-menores, promoviendo una transformación urbana facilitadora de desplazamientos físicamente activos y movimiento seguro al aire libre, teniendo como puntos clave parques, espacios deportivos y centros educativos, mejorando su aprovechamiento para la práctica de AFD saludable y lúdica, extendiéndola a sus vías de conexión según la mirada femenina de Lefaivre (2015).

- Promover programas conciliadores de AFD en el trabajo y tele-empleo, presenciales y virtuales, desarrollados por el propio centro o mediante convenios con organizaciones de la AFD.

- Implementar las ayudas solicitadas, como el reconocimiento de los servicios profesionales de AFD como actividades esenciales y bajo los mismos parámetros o la reducción de su IVA al 4\%.

\section{Referencias bibliográficas}

Castañeda-Babarro, Arkaitz, Arbillaga-Etxarri, Ane, Gutiérrez-Santamaría, Borja y Coca, Aitor. 2020. "Cambio de actividad física durante el encierro por COVID-19". Int J Environ Res Salud Pública 17(18): 6878. doi: 10.3390 / ijerph17186878

Consejo General de la Educación Física y Deportiva. 2020a. La labor de los COLEF autonómicos en la crisis del COVID-19. Acceso 15 de noviembre.

https://www.consejo-colef.es/post/covid19-clipping-colefs

Consejo General de la Educación Física y Deportiva. 2020b. "Manifiesto por la consideración como actividad esencial de la EF, la AF y el deporte". Acceso 15 de noviembre. https://www.consejo-colef.es/post/ covid19-iva

Consejo Superior de Deportes-Federación Española de Municipios y Provincias. 2011. Guía para la incorporación de la perspectiva de género a la gestión deportiva local. Madrid: CSD-FEMP. https://sede. educacion.gob.es

Council of Europe. 2019a. ALL IN: Towards gender balance in sport (Erasmus +). Toolkit | How to Make an Impact on Gender Equality in Sport 4 - Drawing up an action plan. Council of Europe. https://rm.coe. int/toolkit-factsheet-4-drawing-up-an-action-plan-toachieve-gender-equali/1680971aaa

Council of Europe. 2019b. ALL IN: Towards gender balance in sport (Erasmus +). Toolkit | How to Make an Impact on Gender Equality in Sport 8 - Sporting opportunities. Council of Europe. https://rm.coe.int/toolkit-factsheet-8-sporting-opportunities/1680971aae

Espada, María, Martín, María, Jiménez, Víctor, del Hierro, David, Santacruz, José Antonio y Jímenez-Beatty, José Emilio. 2019. "Organizational aspects of adults' sports habits in Spain: gender and social differences". Motricidade 15(1): 24-32. http://dx.doi. org/10.6063/motricidade.13997

Espada, María, Martín, María, Jiménez, Víctor, Moscoso, David, Jiménez-Beatty, Emilio y Rivero, An- 
tonio. 2018. "Leisure sports habits in Spanish adults: gender and social differences". OBETS. Revista de Ciencias Sociales 13(2): 495-513.doi: 10.14198/ OBETS2018.13.2.02

Fasting, Kari, Sand, Trond Svela, Pike, Elizabeth y Matthews, Jordan. 2014. From Brighton to Helsinki. Women and Sport Progress Report 1994-2014. Valo: International Working Group Women and Sport-Finish Sport Confederation.

García Ferrando, Manuel y Llopis, Ramón. 2011. Ideal democrático y bienestar personal. Encuesta sobre hábitos deportivos en España 2010. Madrid: Consejo Superior de Deportes -Centro de Investigaciones Sociológicas. https://libreria.cis.es

García-Tascón, Marta, Sahelices-Pinto, César - Mendaña-Cuervo, Cristina y Magaz-González, Ana María. 2020. “El impacto del encierro por COVID-19 en los hábitos de práctica de AF según género (hombre / mujer): caso español". Int J Environ Res Salud Pública 17(19): 6961. doi: 10.3390 / ijerph17196961

Instituto Nacional de Estadística. 2020. Demografía y población.

https://Www.ine.es/dyngs/INEbase/es/categoria. htm?c=Estadistica_P\&cid=1254735572981

Instituto de la Mujer y para la Igualdad de oportunidades. 2020a. La perspectiva de género, esencial en la respuesta a la COVID-19. Madrid: Ministerio de Igualdad - Instituto de la Mujer y para la Igualdad de oportunidades. https://www.inmujer.gob.es

Instituto de la Mujer y para la Igualdad de oportunidades. 2020b. Mujeres en Cifras-Conciliación-Usos del tiempo. https://www.inmujer.gob.es

International Working Group on Women and Sport. 2020. "ALERT: IWG responds to COVID-19 with global 'Call to Action'". Acceso 15 de noviembre. https://iwgwomenandsport.org/es/iwg-responds-to-covid-19pandemic-with-global-call-to-action/

Lefaivre, Liane. 2015. Gound-up city play as a design tool. Rotterdam: 010 Publishers.

López Martínez, Juan José, Rodríguez-Roiz Juan y Salcedo Cánovas, César. 2020. Lesiones musculoesqueléticas secundarias al ejercicio durante el encierro por la pandemia COVID-19. Med Clin 155 (5): 221-222.doi: 10.1016 / j.medcle.2020.05.013

Martín, María, Barriopedro, María Isabel y Espada, María. 2020. "Influencia de la edad, la crianza y el empleo en las barreras para la práctica de ejercicio físico y deporte de las mujeres adultas en España". En Actas I Congreso Internacional de deporte, salud y emprendimiento (En prensa). Universidad de Granada y Universidad de Baja California.

Martín, María, Espada, María y Barriopedro, María Isabel. 2020. "La demanda y modo de práctica de las personas adultas madrileñas pre y post subida del
IVA de los servicios deportivos: Diferencias de género". En Actas I Congreso Internacional de deporte, salud y emprendimiento (En prensa). Universidad de Granada y Universidad de Baja California.

Martín, María (dir.), Martínez del Castillo, Jesús, Fasting, Kari, Barriopedro, Maribel, Jiménez-Beatty, José Emilio y Rivero, Antonio. 2014. Proyecto $1+D+i$ Necesidades, barreras e innovaciones en la oferta deportiva a las mujeres adultas en España: Informe para profesionales y organizaciones deportivas. Madrid: Grupo de Investigación psicosocial en el deporte Universidad Politécnica de Madrid. http://investigacionsocialdeporte.com

Martínez del Castillo, Jesús, Jiménez-Beatty, José Emilio, Graupera, José Luis, Martín, María, Campos, Antonio y del Hierro, David. 2010. "Being physically active in old age: relationships with being active earlier in life, social status and agents of socialisation". Ageing \& Society 30(7): 1097-1113. https://doi. org/10.1017/S0144686X10000358

Ministerio de Cultura y Deporte. 2020. Anuario de estadísticas deportivas 2020.Madrid: Secretaría General Técnica-Ministerio de Cultura y Deporte. https:// www.culturaydeporte.gob.es

Moscoso-Sánchez, David. 2020. "El contexto del deporte en España durante la crisis sanitaria de la CovID-19". Sociología del Deporte 1(1): 15-19. doi: http://doi.org/10.46661/socioldeporte.5000

Moscoso-Sánchez Sánchez, David y Moyano Estrada, Eduardo (coords.), Biedma-Velázquez, Lourdes, Fernández-Ballesteros-García, Rocío, Martín-Rodríguez, María, Ramos-González, Carlos, Rodríguez-Morcillo-Baena, Luis y Serrano del Rosal, Rafael. 2008. Deporte, salud y calidad de vida. Barcelona: Fundación Obra Social La Caixa.

Moscoso-Sánchez, David y Rodríguez-Díaz, Álvaro. 2020. "El sesgo de la participación deportiva en España: argumentos y análisis alternativos". En Diálogos sobre el deporte (1975-2020), 15 - 32. Barcelona: Generalitat de Catalunya, Secretaria General de I'Esport, Institut Nacional d'Educació Física de Catalunya, Observatori Català de l'Esport.

Piedra, Joaquín. 2020. "Redes sociales en tiempos del COVID-19: el caso de la actividad física". Sociología del Deporte 1(1): 15-19. doi: http://doi.org/10.46661/ socioldeporte.4998

Sánchez-García, Raúl. 2020. "La reutilización lúdica del espacio urbano en tiempos de la COVID-19". Sociología del Deporte 1(1): 25-28. DOl: http://doi. org/10.46661/socioldeporte.4999.

VALGO-Federación Nacional de Empresarios de Instalaciones Deportivas. 2020. Informe Impacto COVID-19 en Instalaciones Deportivas Mayo 2020. Madrid: VALGO-FNEID. https://valgo.es 